Check for updates

Cite this: RSC Adv., 2019, 9, 12864

\title{
Effect of structure regulation of hyper-branched polyester modified carbon nanotubes on toughening performance of epoxy/carbon nanotube nanocomposites
}

\author{
Lu Li, ${ }^{\text {abc }}$ Xia Liao, (D) *a Xingyue Sheng, ${ }^{\mathrm{b}}$ Zengheng Hao, ${ }^{\text {bc }}$ Leilei He, ${ }^{\mathrm{b}}$ Pan Liu, ${ }^{\mathrm{b}}$ \\ Hongbin Quan ${ }^{\mathrm{b}}$ and Yi Zhang ${ }^{\mathrm{b}}$
}

In this paper, carboxylic multi-walled carbon nanotubes (MWCNTs-COOH) were modified by a series of hyperbranched polyesters (HBP) with different molecular structures (different branching degree) through surface grafting, and then the epoxy resin (EP)/carbon nanotube composites were prepared to explore the influences of structure regulation of HBP modified carbon nanotubes on the toughening performance of the composites. The results of Fourier transform infrared spectroscopy (FTIR), transmission electron microscopy (TEM), X-ray photoelectron spectroscopy (XPS), and thermogravimetric analysis (TGA) of various HBP grafted carbon nanotubes confirmed that the HBP were successfully grafted onto MWCNTs- $\mathrm{COOH}$ via an esterification reaction between the carboxyl groups of MWCNTs- $\mathrm{COOH}$ and the hydroxyl groups of HBP, meanwhile, the higher the branching degree of the $\mathrm{HBP}$, the higher its grafting ratio onto carbon nanotubes. Furthermore, the outcome of dynamic thermal mechanical analysis (DMA) indicated that the addition of MWCNTs- $\mathrm{COOH}$ increased the storage modulus and glass transition temperature $\left(T_{\mathrm{g}}\right)$ of the pure EP, and surface grafting of various HBP onto MWCNTs- $\mathrm{COOH}$ decreased the $T_{\mathrm{g}}$ and peak height of mechanical loss of composites. And as the branching degree of HBP increased, the interfacial bonding between MWCNTs and the EP matrix became stronger. The results of mechanical performance and morphology analysis also revealed that the addition of HBP grafted MWCNTs- $\mathrm{COOH}$ significantly improved its dispersion and interfacial bonding in the EP matrix, resulting in better performance in the enhancement of toughness of the composites. In addition, it was found that the higher the branching degree of HBP, the better the toughening performance of the composites.

rsc.li/rsc-advances

\section{Introduction}

Epoxy resin, the most commonly used resin in polymer composites, ${ }^{\mathbf{1}, \mathbf{2}}$ has been widely applied in bridge deck pavement materials, binders, aerospace, and electronic composite materials and in other fields for its excellent mechanical properties such as adhesion, and thermal and chemical corrosion resistance. ${ }^{3,4}$ However, the application of epoxy resin-based materials has been greatly limited due to their high crosslinking degree, brittleness, poor impact resistance and easy cracking after curing. ${ }^{5,6}$ Therefore, toughening and modification of epoxy resin has become the focus of current research. ${ }^{5,7-9}$

${ }^{a}$ College of Polymer Science and Engineering, State Key Laboratory of Polymer Materials Engineering, Sichuan University, Chengdu 610065, Sichuan, China. E-mail:xliao@scu.edu.cn

${ }^{b}$ Chongqing Zhixiang Paving Technology Engineering Co., Ltd., Chongqing 401336, China.E-mail: haozengheng@cmhk.com

'China Merchants Chongqing Communications Research \& Design Institute Co., Ltd., Chongqing 400067, China
Toughness, achieved through various deformation mechanisms before failure occurs, implies energy absorption. In past years, great efforts have been made to improve the toughness of epoxy thermosets. One effective mechanism to increase toughness is the addition of a second phase (elastomers), however, at the expense of its other performance such as thermal resistance and strength..$^{5,7,9-11}$

Through the introduction of inorganic fillers to improve the toughness of epoxy resin without sacrificing other properties has been found recently., ${ }^{\mathbf{9 1 2}-14}$ The multi-walled carbon nanotubes (MWCNTs) are ideal fillers for polymer composites due to their high Young's modulus as well as good electrical and thermal conductivity. ${ }^{5,8,15-17}$ Once added with a small amount of MWCNTs, the electrical, thermal and mechanical properties of the polymer matrix can be strongly improved, resulting the composites with good processability, such as balanced stiffness, toughness and other functional properties. However, due to their insolubility, non-melting, easy to tangle and agglomerate, and lack of surface functional groups, carbon nanotubes are 
difficult to process, as well as poor dispersibility and stability, poor compatibility with epoxy matrix materials limiting their application areas. ${ }^{\mathbf{1 4 , 1 8 - 2 1}}$ An effective way to improve the dispersion and interfacial bonding properties of carbon nanotubes in epoxy matrix is surface chemical modification of carbon nanotubes..$^{5,13,15,22}$

Jin et al. ${ }^{23}$ acidified P-MWCNTs (pure carbon nanotubes) to obtain A-MWCNTs (acidified carbon nanotubes). Then AMWCNTs were amine treated (D-MWCNTs) and added to bisphenol A epoxy resin (DGEBA). DGEBA/MWCNTs matrix composites were prepared. Compared with pure DGEBA in mechanical properties, it was found that the interface between MWCNTs and DGEBA was well bonded after modification, which effectively improved the interface between MWCNTs and DGEBA. The dispersibility in bulk resin improves the fracture toughness of DGEBA. Spitalsky et al. ${ }^{24}$ also used acidification and amination treatment, and obtained similar conclusions. However, the traditional acidification and amination are relatively simple surface chemical modification, which makes it difficult to quantitatively control the surface groups of MWCNTs, and the damage to the structure of MWCNTs is obvious. Therefore, it is of great significance to find new surface modification methods for MWCNTs in toughening epoxy resins.

Hyperbranched polymers have a lower branching efficiency than dendrimers while possessing many useful properties. ${ }^{5,6,25,26}$ The highly branched architecture minimizes chainchain ability and low melt viscosity to these polymers, which is compatible with epoxy thermosets. The presence of plenty of functional groups $(-\mathrm{OH})$ provides high reaction activity with inorganic filler, providing us a possibility to be desirable macromolecular surface modifier for MWCNTs, to further enhance the compatibility and dispersion in epoxy resin. On the other hand, research on toughening epoxy resin with hyperbranched polymers is also increasing. J. Yang ${ }^{27}$ used hyperbranched polymer H30 as toughening agent of epoxy resin, the results showed that $\mathrm{H} 30$ can significantly improve the tensile properties, ductility and impact toughness of epoxy resin at low temperature. Jorg Frohlich ${ }^{28}$ developed a new type of epoxy resin with polyether core-shell hyperbranched structure. The results of tensile test, impact toughness and DMA test showed that the toughness of epoxy resin was significantly improved.

Therefore, we believe that the preparation of hyperbranched polymer grafted carbon nanotubes modified epoxy resin can not only improve the dispersion and interfacial bonding of carbon nanotubes (MWCNTs) in epoxy resin (EP) matrix by using a large number of active end groups of hyperbranched polymer, but also further toughen epoxy resin on the basis of EP/ MWCNTs. In the previous study, ${ }^{5}$ we had successfully prepared hyperbranched polyester (H202) grafted MWCNTs, and had applied it in the toughening of epoxy resin cured by diethyltoluene diamine. Results showed that the hyperbranched polyester grafted MWCNTs received a better dispersion in the epoxy prepolymer compared with the raw MWCNTs, which further decreased the glass transition temperature of the EP/MWCNTs composites and exhibited better performance in the enhancement in toughening EP.
Novel method to prepare high performance EP/MWCNTs composites had been provided in the previous study, achieving only investigating one kind of molecular structure of hyperbranched polyester grafted MWCNTs in the system. Still the surface modification of MWCNTs using hyperbranched polyester with different molecular structure might greatly influence the toughening performance, which has not been investigated before. This study aims to comparatively explore the role of hyperbranched polyesters with different branching degrees surface modification of MWCNTs in the toughening performance of the EP/MWCNTs composites.

\section{Experimental}

\subsection{Materials}

The carboxylic multiwalled carbon nanotube (denoted as MWCNTs-COOH in this paper) was purchased from Chengdu Organic Chemicals Co., LTD., Chinese Academy of Science, China. The diameter of the raw MWCNTs-COOH ranged from 10 to $20 \mathrm{~nm}$ and their length was about 5-15 $\mu \mathrm{m}$.

Hyperbranched polyester H201 $\left(M_{\mathrm{w}}=600 \mathrm{~g} \mathrm{~mol}^{-1}\right.$, hydroxyl number $=550 \mathrm{mg} \mathrm{KOH} / \mathrm{g}$ polymer $), \mathrm{H} 202\left(M_{\mathrm{w}}=1200 \mathrm{~g} \mathrm{~mol}^{-1}\right.$, hydroxyl number $=520 \mathrm{mg} \mathrm{KOH} / \mathrm{g}$ polymer $), \mathrm{H} 203\left(M_{\mathrm{w}}=2600 \mathrm{~g}\right.$ $\mathrm{mol}^{-1}$, hydroxyl number $=500 \mathrm{mg} \mathrm{KOH} / \mathrm{g}$ polymer$), \mathrm{H} 204\left(M_{\mathrm{w}}\right.$ $=5400 \mathrm{~g} \mathrm{~mol}^{-1}$, hydroxyl number $=490 \mathrm{mg} \mathrm{KOH} / \mathrm{g}$ polymer was purchased from Wuhan Hyperbranched Polymer Resins Science \& Technology Co. Ltd. (China), and was used as received. Diglycidyl ether of bisphenol A (DGEBA, KE-133L, Chengdu Kemmett Technical Co., Ltd.) (epoxide equivalent: $182 \mathrm{~g} \mathrm{~mol}^{-1}$ ) was dried under vacuum at $80{ }^{\circ} \mathrm{C}$ for $8 \mathrm{~h}$ before use. Diethyltoluene diamine (DETDA) and $N, N$-dimethylformamide (DMF) were purchased from Sinopharm Chemical Reagent Beijing Co., Ltd., China, and were used as received.

The chemical structures of the MWCNTs-COOH and Hyper H201, H202, H203, H204 are shown in Scheme 1.

2.1.1 Grafting of the MWCNTs-COOH. Direct grafting method was applied in this study to obtain various hyperbranched polyester grafting MWCNTs. Firstly, dissolve various hyper-branched polyester (Hyper H201, H202, H203, H204) and MWCNTs-COOH in DMF respectively at room temperature before being irradiated for $1 \mathrm{~h}$ by ultrasound. The mixed systems were heated up to $120{ }^{\circ} \mathrm{C}$ under the oil bath with magnetic stirring for $24 \mathrm{~h}$. Later, they were waited to cool to room temperature about $2 \mathrm{~h}$ for the filtration with PVDF membrane of $0.22 \mathrm{~mm}$ diameter pore. In order to eliminate the unreacted hyper-branched polyester and extra solvent, the product was firstly washed by large amount of acetone and filtered for several times. And the washing stopped only until the washed acetone has the same FT-IR spectra compared with pure acetone while taking washed acetone for FT-IR analysis each time. Then collect the membrane filtrate materials and heat them under vacuum drier at $80{ }^{\circ} \mathrm{C}$ for $24 \mathrm{~h}$. The final products, MWCNTs grafted with various hyperbranched polyester (named as MWCNTs-H201, MWCNTs-H202, MWCNTsH203, and MWCNTs-H204) were obtained eventually. Taking MWCNTs-H201 as an example, the reaction principle between 


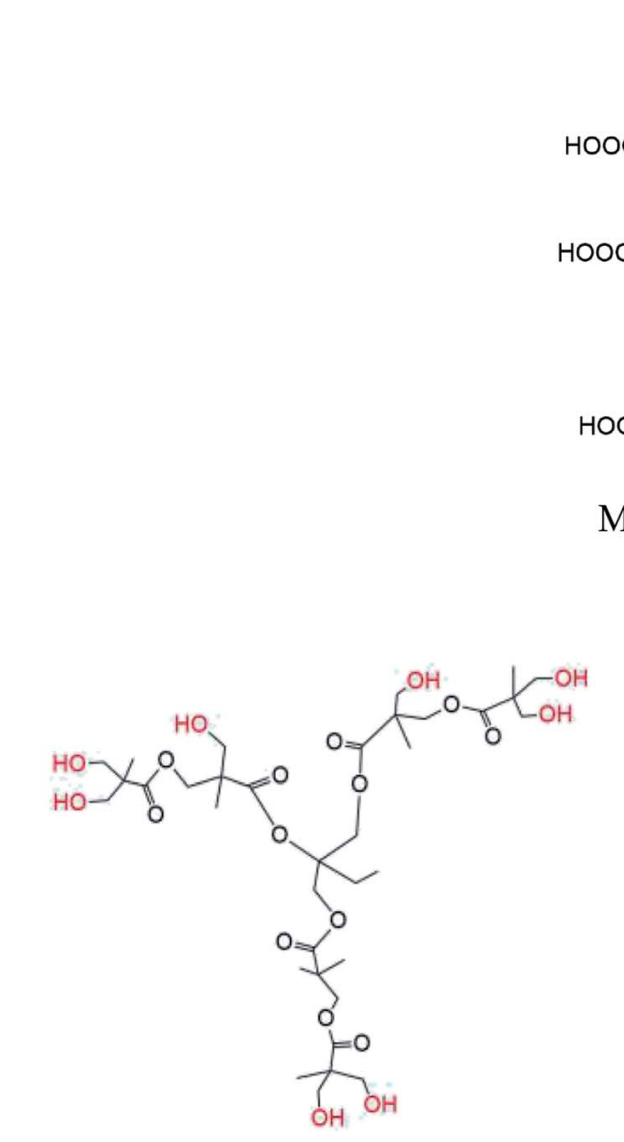

Hyper H201

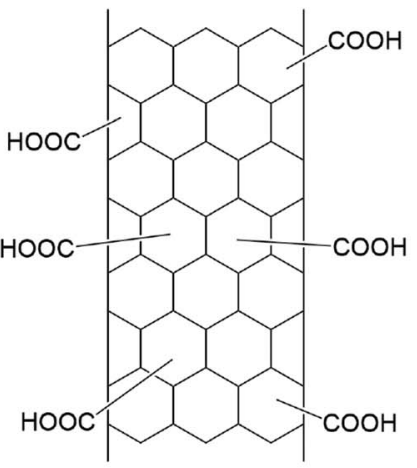

MWCNTs-COOH

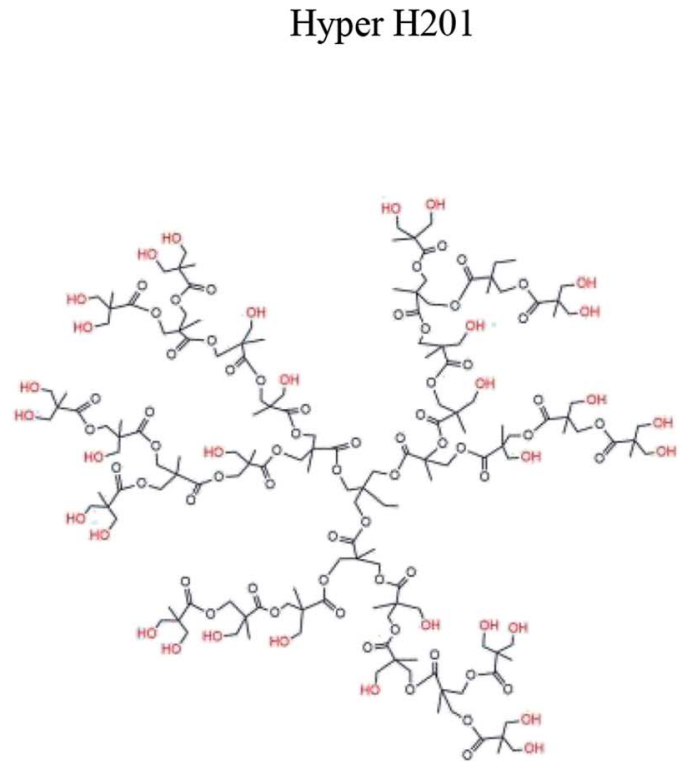

Hyper $\mathrm{H} 203$

(coOH and hyperbr

Scheme 1 Structure of MWCNTs- $\mathrm{COOH}$ and hyperbranched polyester.

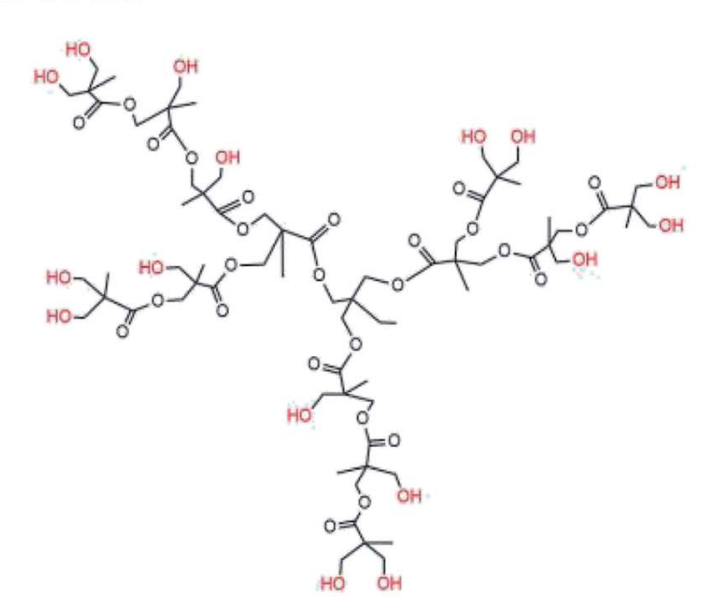

\section{Hyper H202}

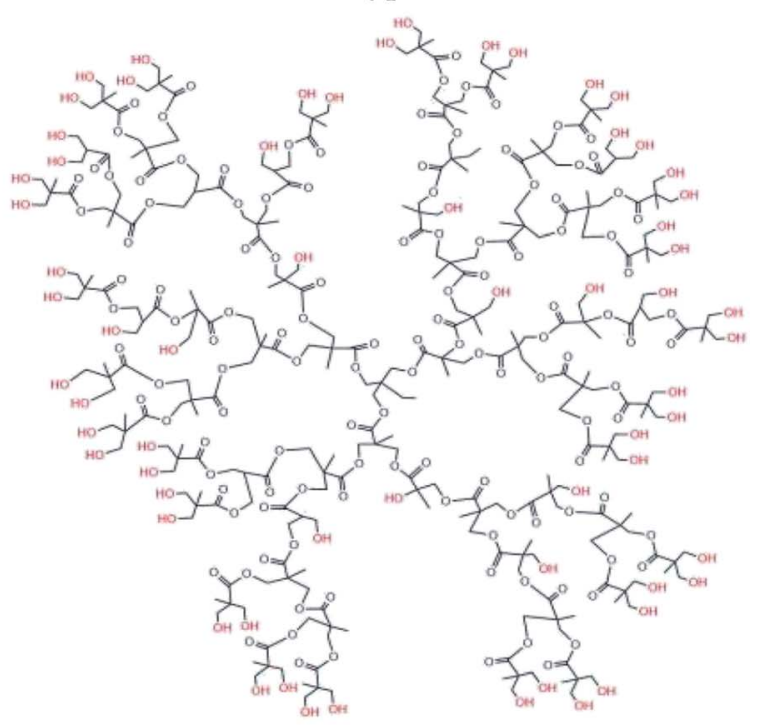

Hyper H204 hyper-branched polyester and MWCNTs-COOH was shown in Scheme 2.

2.1.2 Preparation of EP/MWCNTs-COOH and EP/grafting MWCNTs composites. Firstly, mix epoxy prepolymer and fillers (MWCNTs-COOH or grafting MWCNTs) in the weight ratio of $99: 1$ and then irradiate them using ultrasound for $1 \mathrm{~h}$ to ensure a fine dispersion of MWCNTs-COOH in the epoxy prepolymer. Then, add $20 \mathrm{wt} \%$ DETDA to mix and heat the mixture to $120^{\circ} \mathrm{C}$ for $2 \mathrm{~h}$. The final cooled and cured products in room temperature were named as EP, EP/MWCNTs-COOH, EP/ 

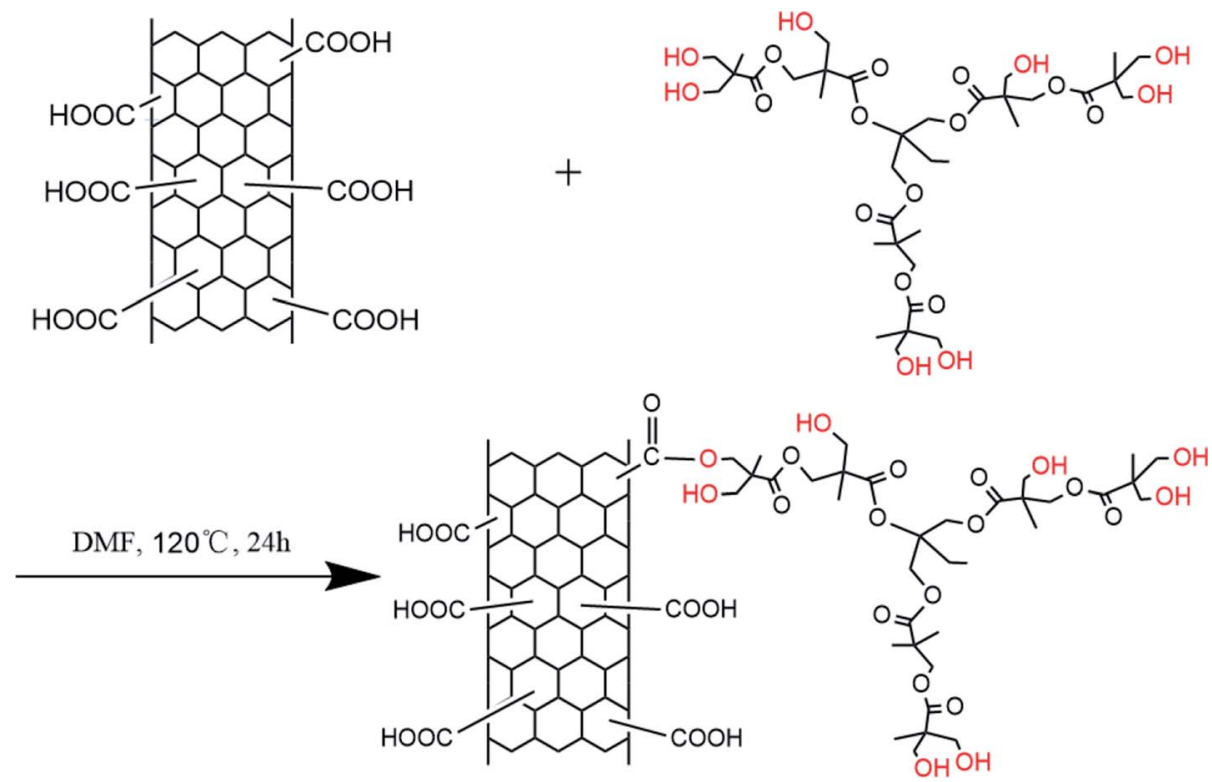

Scheme 2 Reaction principle of hyperbranched polyester modified carbon nanotubes, taking H201 as an example.

MWCNTs-H201，EP/MWCNTs-H202，EP/MWCNTs-H203， EP/ MWCNTs-H204, respectively.

\subsection{Characterization}

2.2.1 Fourier transform infrared spectroscopy (FT-IR). The FT-IR spectra of the samples were tested by a Nicolet iS50 FT-IR spectrometer at a resolution of $4 \mathrm{~cm}^{-1}$ and averaged 20 scans for each spectrum with the scanning range of $4000-400 \mathrm{~cm}^{-1}$.

2.2.2 Transmission electron microscopy (TEM). To study the morphologies of the MWCNTs-COOH and various grafting MWCNTs, transmission electron microscopy (TEM, Tecnai G2 F20, FEI, USA) was applied.

2.2.3 X-ray photoelectron spectroscopy (XPS). X-ray photoelectron spectroscopy analysis was performed with a Kratos Axis Ultra DLD Photoelectron spectrometer utilizing a monochromatized $\mathrm{Al} \mathrm{K} \alpha$ radiation $(1486.6 \mathrm{eV}$ ) as the $\mathrm{X}$-ray source.

2.2.4 Thermogravimetric analysis (TGA). All TGA analyses were conducted with a GENERAL V4.1C DUPONT 2100 thermal analyzer, using a constant heating rate of $20{ }^{\circ} \mathrm{C} \mathrm{min}^{-1}$ from room temperature to $800{ }^{\circ} \mathrm{C}$. All the experiments were undertaken in a nitrogen atmosphere at a purge rate of $50 \mathrm{ml} \mathrm{min}{ }^{-1}$.

2.2.5 Dynamic thermo-mechanical analysis (DMA). In three-point-bending mode, ${ }^{29}$ the dynamic thermo-mechanical analysis was obtained with a DMA Q800 analyzer (TA Corporation, USA), and using the specimen size of $35 \mathrm{~mm} \times 12.8 \mathrm{~mm} \times$ $3.22 \mathrm{~mm}$. The frequency of oscillation was $10 \mathrm{~Hz}$, the temperature ranged from $30{ }^{\circ} \mathrm{C}$ to $150{ }^{\circ} \mathrm{C}$, the heating rate was $2{ }^{\circ} \mathrm{C} \min ^{-1}$ and the deformation amplitude was $20 \mu \mathrm{m}$.

2.2.6 Scanning electronic microscopy (SEM). Firstly, the fractured surface of the samples was cleaned and gold-coated before the SEM (scanning electronic microscopy) analysis. ${ }^{29-31}$ And then the morphology observation (SEM) was performed on a JSM-5900 LV environmental scanning electron microscope (JEOL, Corp. Ltd. Japan) at an accelerating voltage of $20 \mathrm{kV}$.
2.2.7 Mechanical properties. The tensile strength test was conducted using a universal tensile testing machine (Instron 4302, USA), according to the ISO-527-5 standard; Charpy impact strength (known as the Charpy V-notch test) was conducted according to the ISO-179 standard; and Barcol hardness was tested by the ASTM D-2583 standard..$^{32,33}$ At last, the average of the five readings contributed to the reported data.

\section{Results and discussions}

\subsection{Characterization of surface modification of the MWCNTs-COOH}

3.1.1 FT-IR analysis. The FT-IR spectra of hyper-branched polyesters with different branching degrees (Hyper H201, H202, H203, and H204) are shown in Fig. 1a. As can be seen from Fig. 1a, the infrared spectra of hyperbranched polyesters with different branching degrees are very close because of their similar molecular structures. The absorption peak at wave number $3420.9 \mathrm{~cm}^{-1}$ represents the stretching vibration of hydroxyl groups in the molecular structure. ${ }^{34,35}$ The absorption peak at wave numbers $2921.9 \mathrm{~cm}^{-1}$ and $2888.5 \mathrm{~cm}^{-1}$ represents the antisymmetric stretching vibration, symmetric stretching vibration of methyl and methylene groups respectively. And $1734.4 \mathrm{~cm}^{-1}$ is the stretching vibration peak of carbonyl groups. ${ }^{36-38}$

For FT-IR spectra of different branching degrees of hyperbranched polyester modified carbon nanotubes, it can be observed from Fig. 1b, compared with the pre-grafting (MWCNTs-COOH), the absorption peak of carbon nanotubes at the wave numbers of $2921.9 \mathrm{~cm}^{-1}$ and $2888.5 \mathrm{~cm}^{-1}$ were added after four different branching degrees of hyperbranched polyester grafting modifications, corresponding to the antisymmetric and symmetric stretching vibration of methyl and methylene, respectively. And the higher the branching degree of hyperbranched polyester increased, the stronger the absorption 


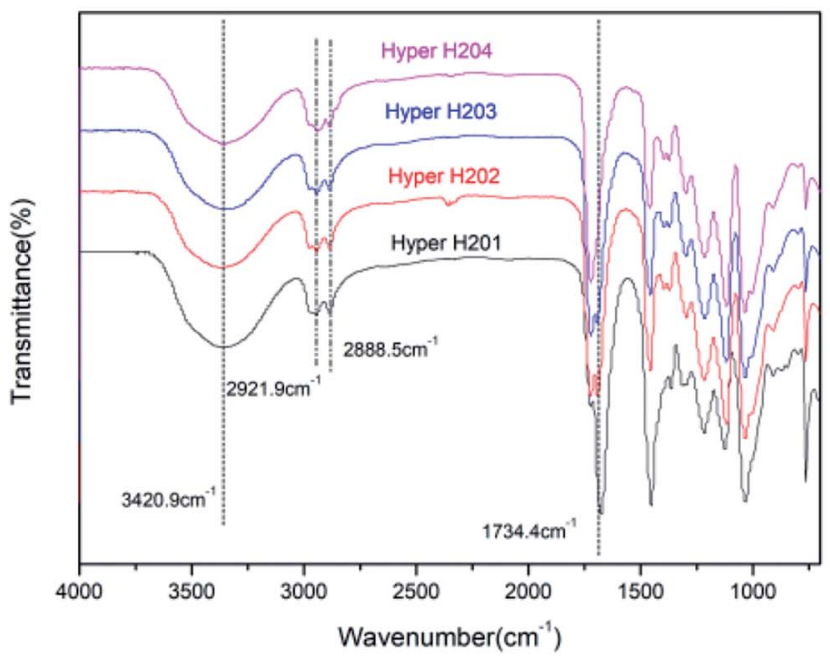

(a)

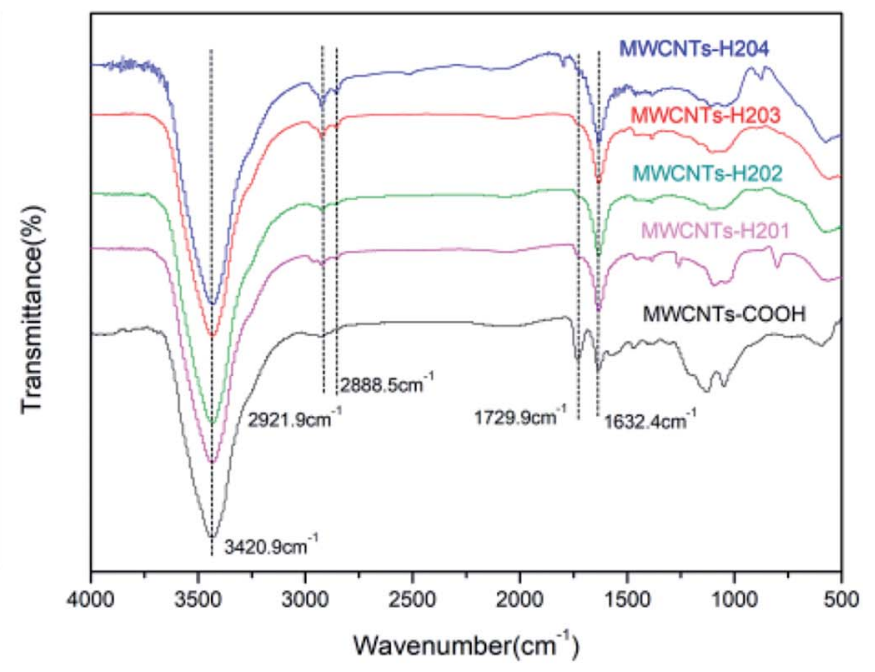

(b)

Fig. 1 FT-IR spectra of (a) hyper-branched polyester with different branching degrees and (b) different grafted MWCNTs-COOH.

peak strength was. Since hyperbranched polyester contains large amounts of methyl and methylene in their molecular structure, it can be inferred from Fig. $1 \mathrm{~b}$ that all four kinds of hyperbranched polyesters with different branching degrees have been successfully grafted onto carbon nanotubes. Moreover, it can also be seen from Fig. $1 \mathrm{~b}$ that the intensity of the absorption peak at $1729.9 \mathrm{~cm}^{-1}$ representing the stretching vibration of carbonyl decreases obviously after grafting. This is because when carbonyl exists in the form of carboxylic acid, the absorption peaks are generally stronger due to hydrogen bonding. When carboxyl groups on carbon nanotubes surface are esterified with hydroxyl-terminated hyperbranched polyesters, the carbonyl absorption peaks will be significantly decreased.

Since the residual hyperbranched polyesters of each group of carbon nanotubes have been washed away by acetone before the infrared spectroscopy test, the above results suggest that the grafting reaction of hyperbranched polyesters of different branched degrees with carboxylated carbon nanotubes has occured, and the reaction principle is the esterification reaction between carboxyl groups on the surface of carbon nanotubes and hydroxyl groups of different hyperbranched polyesters.

3.1.2 TEM analysis. As shown in Fig. 2, TEM images of MWCNTs and various grafting MWCNTs were recorded. It can be seen that the morphologies of MWCNTs and various grafting MWCNTs were quite different from each other. From Fig. 2a, the typical morphological characteristics of carbon nanotubes can be clearly observed. However, from Fig. $2 \mathrm{~b}-\mathrm{e}$, uniform dark morphology can be observed on the surface of various grafting carbon nanotubes, and with the increase of branching degree of hyper-branched polyester, the diameter of grafting carbon nanotubes increases gradually, indicating that the MWCNTs is covered with hyper-branched polyester, and the grafting amount of MWCNTs increases with the increase of the branching degree of hyper-branched polyester.
3.1.3 XPS analysis. In order to further verify the successful grafting of hyperbranched polyesters with different branching degrees onto the surface of carbon nanotubes, the original MWCNTs-COOH and modified MWCNTs-COOH by different hyperbranched polyesters were compared and analyzed by X-ray photoelectron spectroscopy, as shown in the Fig. 3.

As can be seen from Fig. 3, the C1s and O1s peaks of all carbon nanotube samples are shown by X-ray photoelectron spectroscopy. For the original carboxylated carbon nanotubes, the weak O1s peaks are mainly attributed to the oxygen atoms contained in the carboxyl groups on the surface of carbon nanotubes. For hyperbranched polyester modified carbon nanotubes with different branching degrees, the O1s peak increased significantly, and along with the increase of branching degree in the molecular structure of hyperbranched polyester, the intensity of $01 \mathrm{~s}$ peak increased, proving that hyperbranched polyesters with different branching degrees did exist on the surface of carbon nanotubes.

Furthermore, the C1s and O1s fine spectral peaks of each group of samples were analyzed. It can be seen from the fine spectrum of O1s peaks (as shown in Fig. 4b). Compared with unmodified carboxylated carbon nanotubes, the intensity of O1s peaks of different hyperbranched polyester modified carbon nanotubes increased significantly. The order of strength is MWCNTs-H204 > MWCNTs-H203 > MWCNTs-H202 > MWCNTs-H201 > MWCNTs-COOH. This is because the higher the degree of branching of hyperbranched polyester increases, the more hydroxyl end groups are introduced into carbon nanotubes, which gradually enhances its O1s peak strength.

At the same time, it can be seen from the fine spectrum of C1s peak (as shown in Fig. 4a) that the strength order of C1s peak is opposite to that of O1s peak. This phenomenon may be due to a series of chemical treatments in the process of surface hyperbranching of carbon nanotubes. Semi-quantitative X-ray photoelectron spectroscopy analysis of surface elements for 

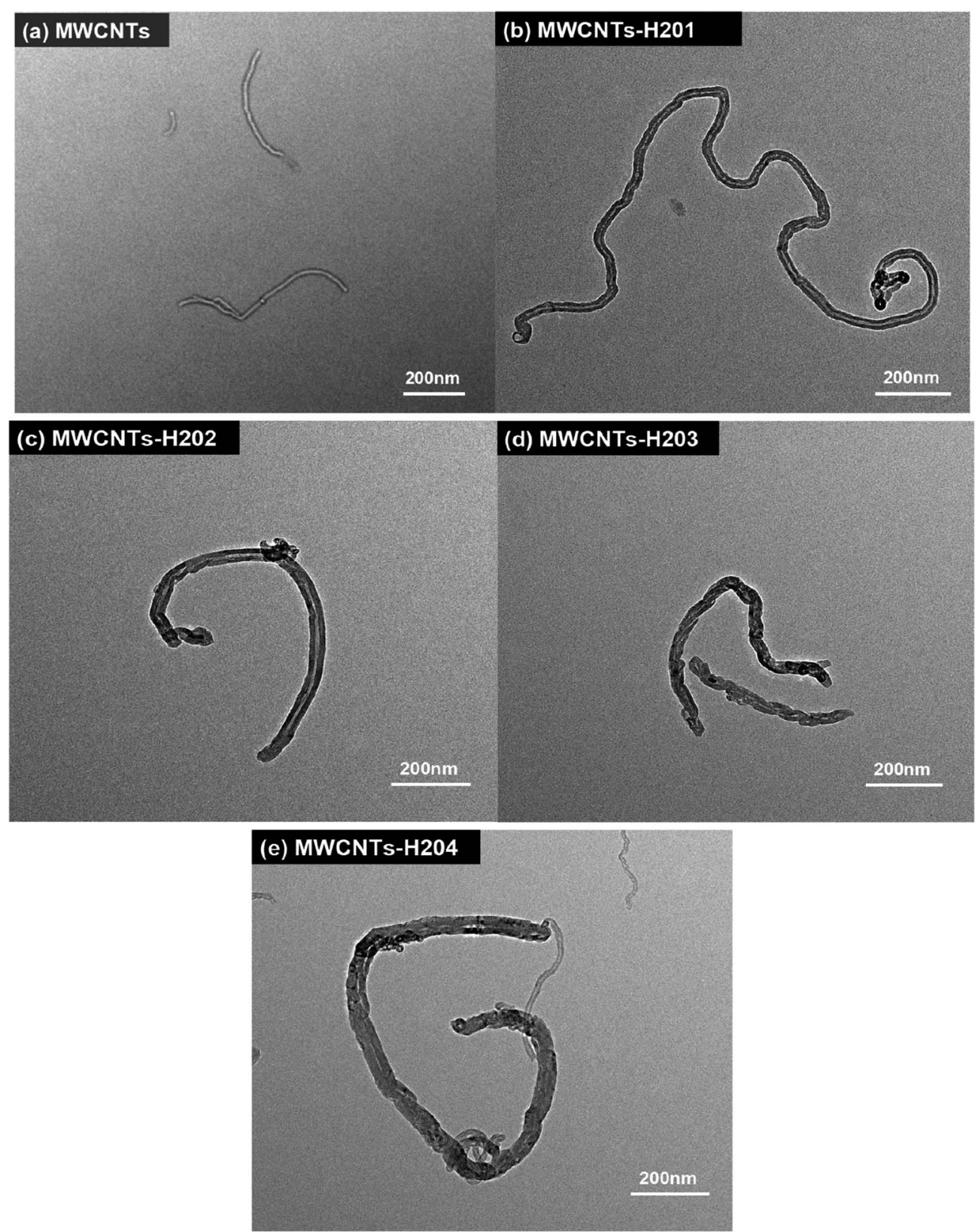

Fig. 2 TEM images of the (a) MWCNTs, (b) MWCNTs-H201, (c) MWCNTs-H202, (d) MWCNTs-H2O3 and (e) MWCNTs-H2O4.

each group of samples is shown in Table 1. From Table 1, it can be seen that the changes of atomic percentage of $\mathrm{C}$ and $\mathrm{O}$ on the surface of hyperbranched polyester modified carbon nanotubes and carboxylated carbon nanotubes with different branching degrees are consistent with the results of qualitative discussion.

To further explore the effect of surface modification of hyperbranched polyester on carbon nanotubes, the C1s peaks were separated according to the binding energy of different valence states of each element, as shown in Fig. 5. For the original carboxylated carbon nanotubes (Fig. 5e), the C1s peak can be decomposed into three single peaks, in which the binding energy $284.6 \mathrm{eV}$ is the main peak formed by $\mathrm{sp}^{2}$ hybrid $\mathrm{C}$ atom in the coiled graphite layer of carboxylated carbon nanotubes, and the binding energy $285.6 \mathrm{eV}$ is the characteristic peak formed by a small number of structural defects in the preparation process of carboxylated carbon nanotubes. The energy $289.0 \mathrm{eV}$ was the characteristic peak of $\mathrm{C}$ atom in $-\mathrm{COOH}$ on the surface of carboxylated carbon nanotubes. The hyperbranched polyester modified carbon nanotubes with different branching degrees showed that a new single peak appeared at the binding energy $286.8 \mathrm{eV}$ of the four groups of samples, which represented the characteristic peak of $\mathrm{C}$ atom contained in the terminal hydroxyl $\mathrm{C}-\mathrm{OH}$ in the molecular structure of hyperbranched polyester.

In conclusion, it is strongly proved that hydroxyl-terminated hyperbranched polyesters with different branching degrees are grafted onto the surface of carbon nanotubes in the form of covalent bonds rather than simple adsorption. 


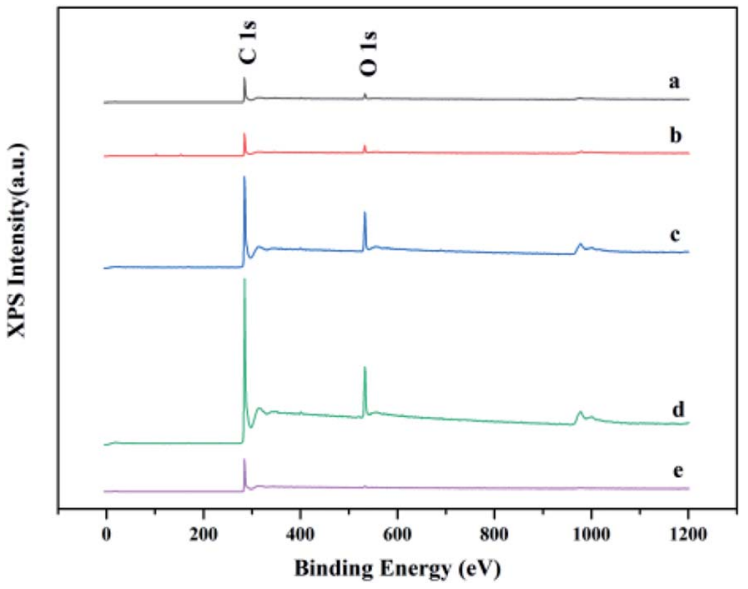

Fig. 3 XPS survey spectra of (a) MWCNTs-H201, (b) MWCNTs-H2O2, (c) MWCNTs-H203, (d) MWCNTs-H204, (e) MWCNTs-COOH.

3.1.4 TGA analysis. The TGA curves of different degrees of hyperbranched polyester are shown in Fig. 6. All four kinds of hyperbranched polyesters with different branching degrees began to decompose at about $300{ }^{\circ} \mathrm{C}$, and the decomposition rate increased sharply with the increase of temperature. In a smaller temperature range, the hyperbranched polyesters basically decomposed completely, and then the TGA curves gradually flattened out. The weight loss rate no longer changed. When the temperature was about $800{ }^{\circ} \mathrm{C}$, the residual mass ratios of hyper $\mathrm{H} 201, \mathrm{H} 202$, $\mathrm{H} 203$ and $\mathrm{H} 204$ were $7.52 \%, 3.15 \%$, $4.28 \%$ and $0.57 \%$, respectively.

The TGA curves of different degrees of hyperbranched polyester modified carbon nanotubes are plotted in Fig. 7.

For MWCNTs-COOH, there was no obvious mass loss in the temperature range of $30-800{ }^{\circ} \mathrm{C}$. A small amount of mass loss was due to the carboxyl functional groups on the surface of carbon nanotubes and a small amount of impurities. However,
Table 1 Percentage content of surface composition of various MWCNTs-COOH samples

\begin{tabular}{llll}
\hline Samples & C1s (\%) & O1s (\%) & $n_{\mathrm{O}} / n_{\mathrm{C}}$ \\
\hline MWCNTs-H201 & 25.06 & 74.94 & 0.33 \\
MWCNTs-H202 & 33.10 & 66.90 & 0.49 \\
MWCNTs-H203 & 34.19 & 65.81 & 0.52 \\
MWCNTs-H204 & 39.66 & 60.34 & 0.66 \\
MWCNTs-COOH & 10.80 & 89.20 & 0.12 \\
\hline
\end{tabular}

when the temperature of MWCNTs-COOH grafted with four different hyperbranched polyesters was increased to $300{ }^{\circ} \mathrm{C}$, all TGA curves showed obvious inflection points and the decomposition rate increased significantly, respectively.

During the preparation of samples, the reaction products were washed repeatedly by acetone, and the unreacted residual hyperbranched polyesters on the surface of carbon nanotubes were removed as far as possible. Therefore, it can be inferred that the rapid decline of TGA curves of each group of samples was due to the grafting of carbon nanotubes. The rapid decomposition of hyperbranched polyesters in this temperature range also proved that hyperbranched polyesters with different branching degrees had been successfully grafted onto carbon nanotubes.

Since the TGA curve directly reflects the grafting amount of hyperbranched polyesters on the surface of carbon nanotubes, the four different hyperbranched polyesters begin to decompose at about $300{ }^{\circ} \mathrm{C}$, and almost completely decompose at about $600{ }^{\circ} \mathrm{C}$. Therefore, the grafting amount of hyperbranched polyesters with different branching degrees on carbon nanotubes can be calculated by the following formula.

$$
F_{\mathrm{wt}}=\Delta M_{\mathrm{H}}-\Delta M_{\mathrm{COOH}}
$$

Among the formula, $F_{\mathrm{wt}}$ is the grafting amount of hyperbranched polyester onto carbon nanotubes, $\Delta M_{\mathrm{H}}$ is

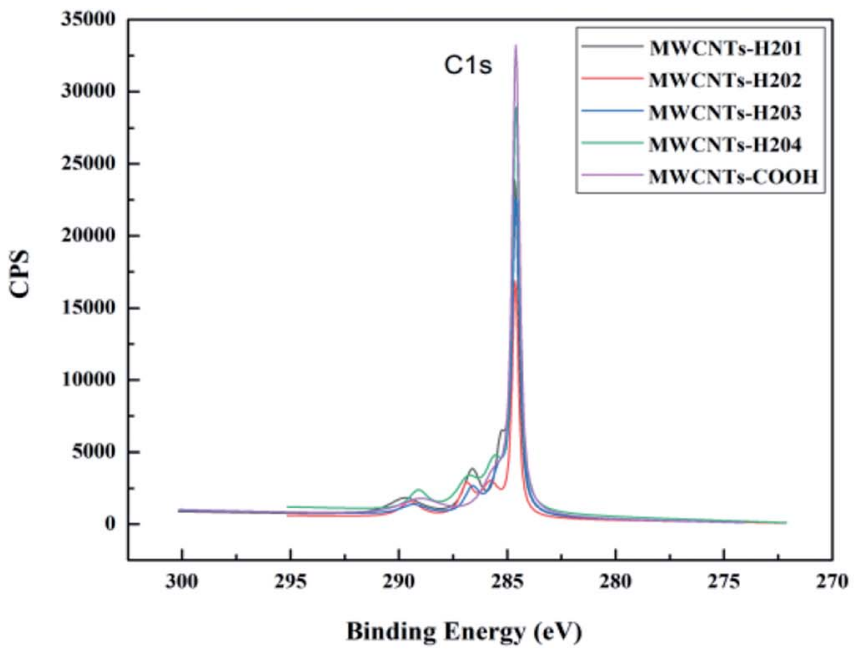

(a)

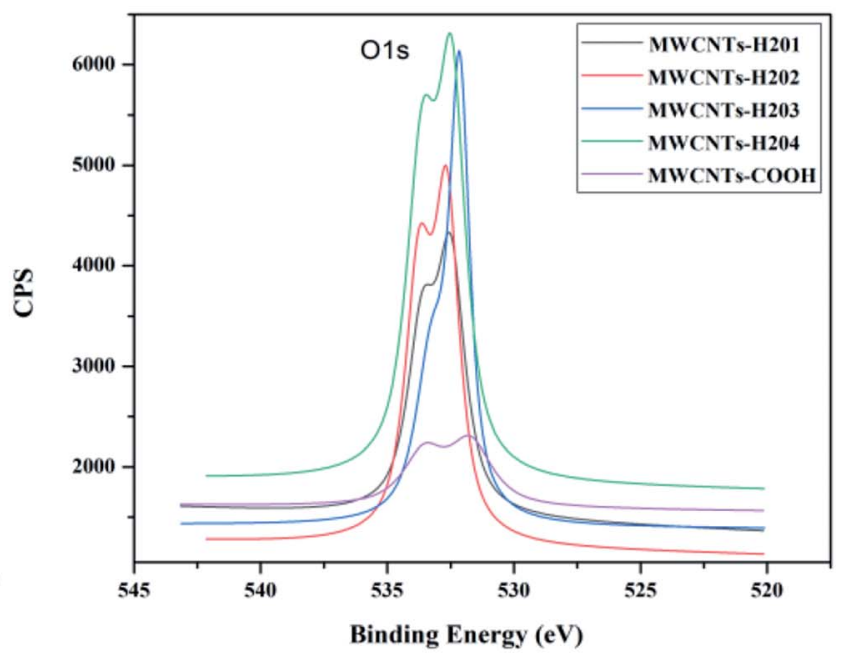

(b)

Fig. 4 High-resolution (a) C1s and (b) O1s XPS spectra of various MWCNTs-COOH samples. 

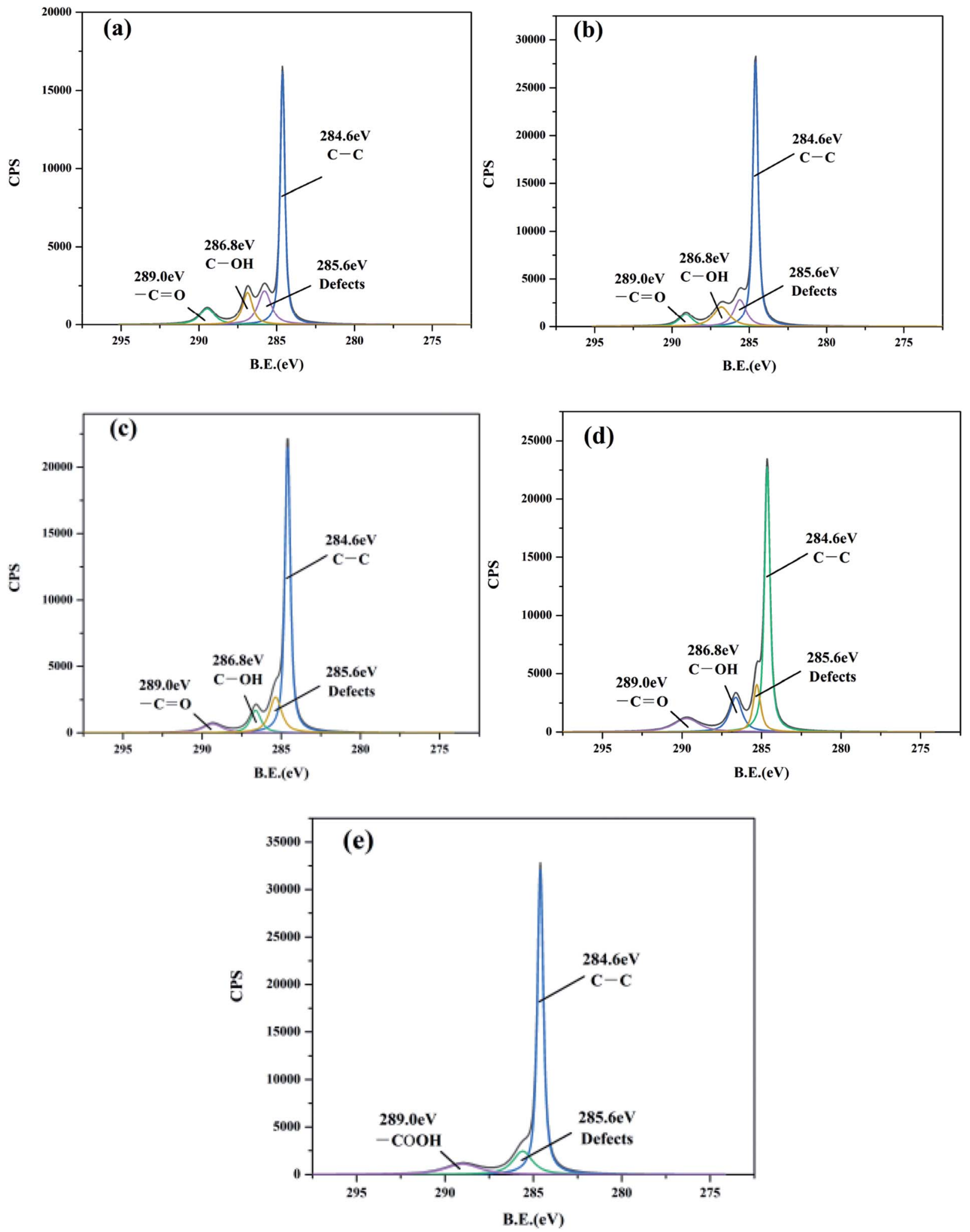

Fig. 5 The C1s peak separation of (a) MWCNTs-H201, (b) MWCNTs-H202, (c) MWCNTs-H203, (d) MWCNTs-H204, (e) MWCNTs-COOH.

the weight loss percentage of hyperbranched polyester modified carbon nanotubes at $600{ }^{\circ} \mathrm{C}$, and $\Delta M_{\mathrm{COOH}}$ is the weight loss percentage of carboxylated carbon nanotubes at $600{ }^{\circ} \mathrm{C}$.
As shown in Table 2, the grafting content of hyperbranched polyesters with different branching degrees on carbon nanotubes was calculated, respectively. 


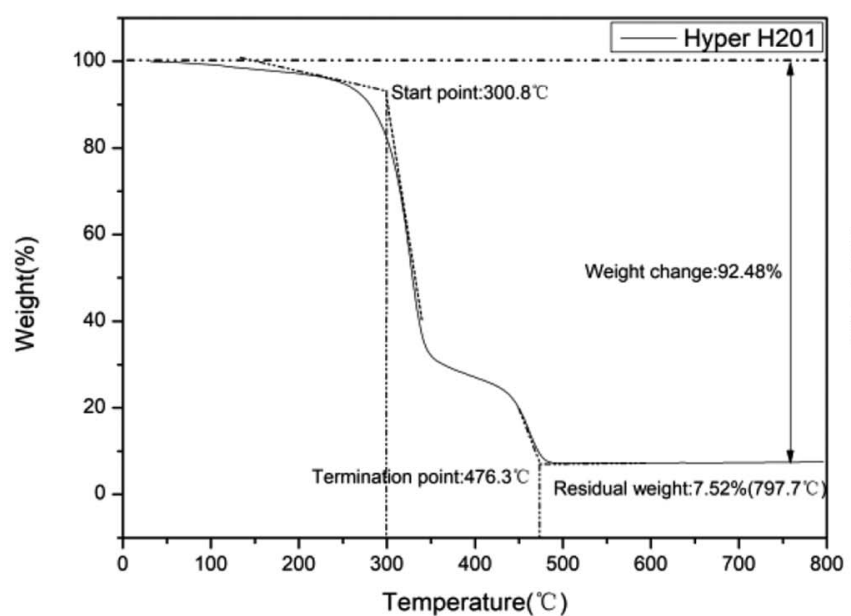

(a)

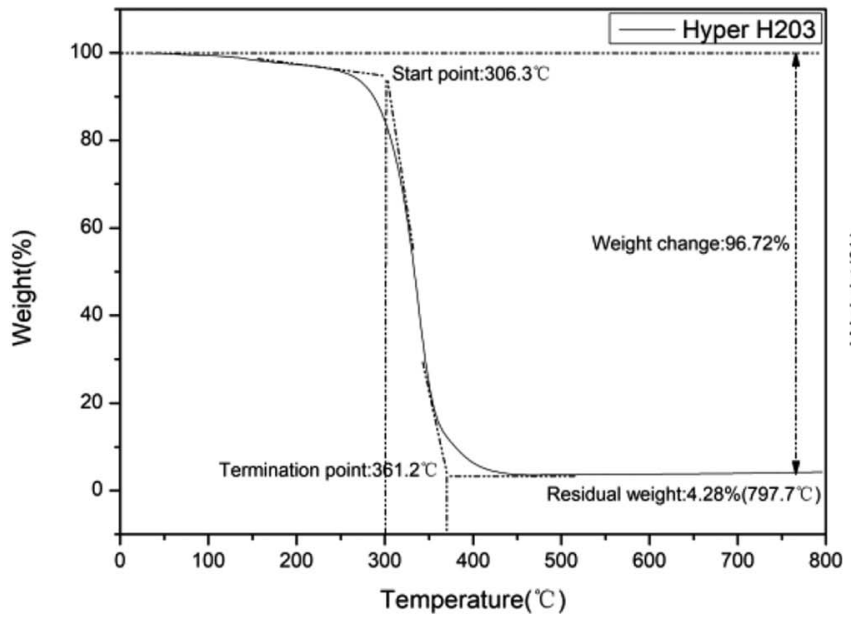

(c)

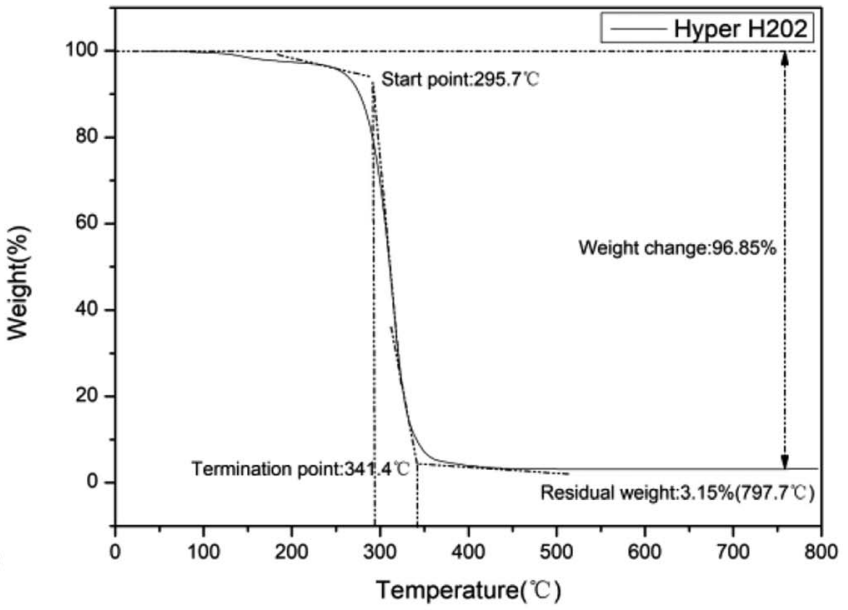

(b)

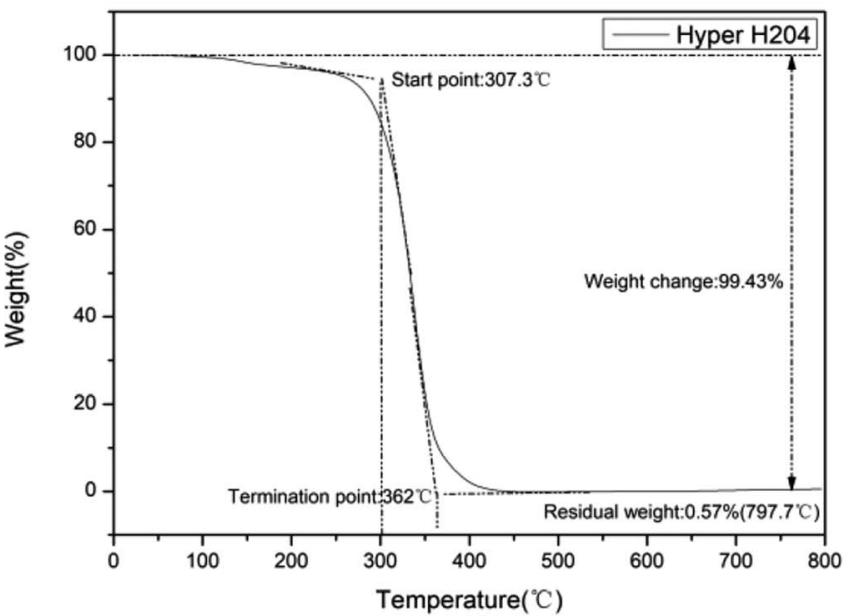

(d)

Fig. 6 TGA curves of (a) Hyper H2O1, (b) Hyper H2O2, (c) Hyper H2O3 and (d) Hyper H2O4.

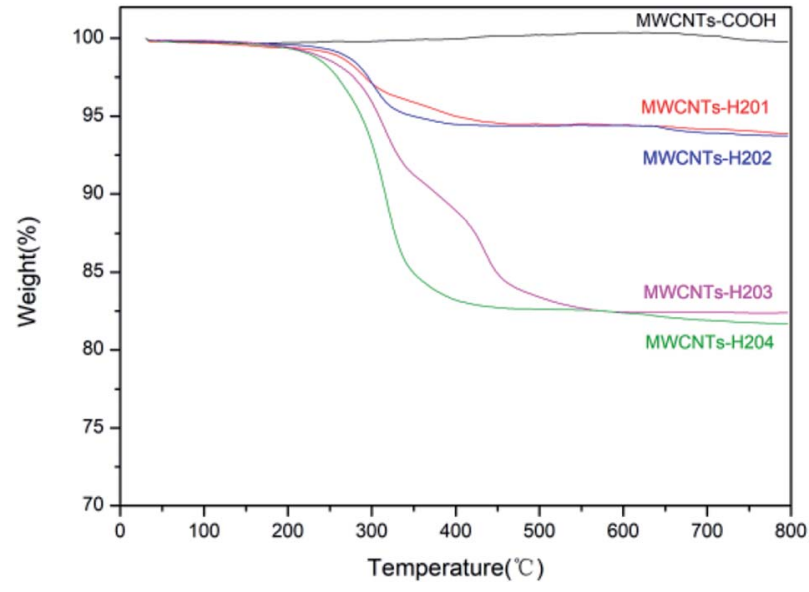

Fig. 7 TGA curves of MWCNTs-COOH, MWCNTs-H201, MWCNTsH2O2, MWCNTs-H2O3, and MWCNTs-H2O4.
Table 2 The grafting content of hyperbranched polyester modified carbon nanotubes

\begin{tabular}{ll}
\hline Samples & $F_{\mathrm{wt}}$ \\
\hline MWCNTs-H201 & $5.90 \%$ \\
MWCNTs-H202 & $6.05 \%$ \\
MWCNTs-H203 & $17.40 \%$ \\
MWCNTs-H204 & $18.10 \%$
\end{tabular}

\subsection{Performances of EP/MWCNTs-COOH composites}

3.2.1 DMA measurement. Carboxylated carbon nanotubes modified epoxy resin and hyperbranched polyester with different branching degrees grafted carbon nanotubes modified epoxy resin were prepared, respectively. The weight ratio of different MWCNTs-COOH to epoxy resin was $0.3 \%$. Then the dynamic mechanical properties were tested and compared with pure epoxy resin system as follows. 
Fig. 8 shows the DMA patterns of the storage modulus to temperature of different epoxy/carbon nanotube composites. It can be seen from the figures that the storage modulus of the composites is obviously improved by adding MWCNTs-COOH without hyperbranched polymer modification compared with pure epoxy resin. This is because MWCNTs-COOH are rigid inorganic carbon nanomaterials with high aspect ratio, high strength and stiffness, and MWCNTs-COOH play a role of physical crosslinking point in epoxy resin, which reduces the mobility of molecular chains, so the storage modulus of epoxy resin filled with carbon nanotubes is greatly increased. However, after modification with hyperbranched polyester, the storage modulus of epoxy composites decrease obviously, and with the increase of the branching degree of hyperbranched polyester, the storage modulus decrease more obviously. The possible reason for this phenomenon is that there are a large number of molecular branched chains in the molecular structure of hyperbranched polyester which distribute in the resin matrix. For the reason that the hyperbranched structure of internal molecular chains does not cross-link, the uncrossed hyperbranched structure acts as a "flexible segment" in the matrix and becomes a part of the epoxy resin cross-linking network ${ }^{39}$ which providing greater mobility for the low-level kinematic units of epoxy resin molecules smaller than chain segments. Thus the storage modulus of epoxy composites decreases, as the higher the branching degree increased, the higher the content of the "flexible segment" and the more obvious the reduction of the storage modulus were.

Fig. 9 shows the DMA spectrum of mechanical loss of epoxy resin/carbon nanotube composites. It can be seen from the diagram that all the tested samples are homogeneous systems with only one glass transition temperature $\left(T_{\mathrm{g}}\right)$. Compared with pure epoxy resin, the glass transition temperature of MWCNTs$\mathrm{COOH}$ without hyperbranched polymer modification increases significantly after adding epoxy resin matrix. However, after the modification of MWCNTs-COOH with hyperbranched polymers, the $T_{\mathrm{g}}$ of the resin matrix decreases significantly, and with

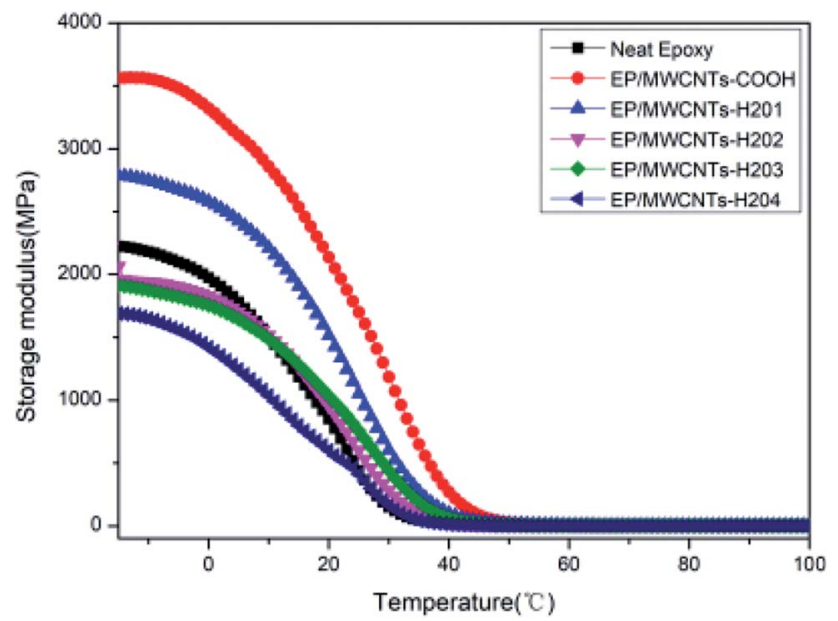

Fig. 8 Storage modulus curves of neat EP and various EP/MWCNTs$\mathrm{COOH}$ composites.

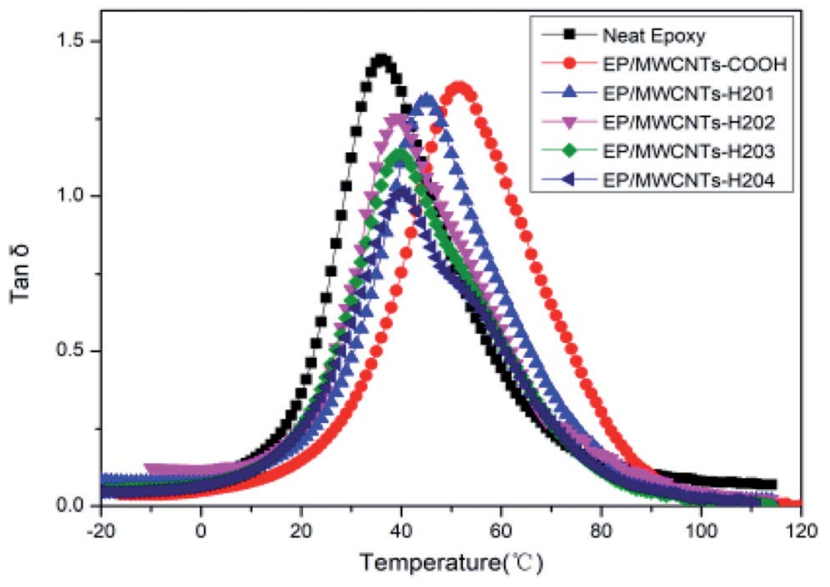

Fig. 9 Mechanical loss curves of neat EP and various EP/MWCNTs composites.

the increase of branching degree, the $T_{\mathrm{g}}$ decreases more obviously, but when the branching degree increases to a certain extent, the $T_{\mathrm{g}}$ value does not change. It is noteworthy that after grafting modification of carbon nanotubes with hyperbranched polymers, the loss peak height also shows a significant downward trend with the increase of branching degree of hyperbranched polymers. This is due to the good compatibility and chemical bonding force between hyperbranched polymers and epoxy resin matrix, ${ }^{39}$ which increases the interfacial bonding between carbon nanotubes and matrix. And with the increase of branching degree, the stronger the interfacial bonding, the more uniformly dispersed MWCNTs-COOH hinder the movement of polymer chains, so the loss peak height is reduced.

3.2.2 Mechanical properties. In this section, the mechanical properties of various epoxy composites at room temperature were studied, which can to some extent reflect the toughness, even at low temperature toughness of the composites. All the weight ratio of different MWCNTs-COOH to epoxy resin was $0.3 \%$. The tensile strength, elongation at break, Charpy impact strength and Barcol hardness are recorded in Table 3.

The tensile strength is the stress at which a material breaks or permanently deforms. As an intensive property, it does not depend on the size of the test specimen. In addition, Charpy impact strength is a standardized high strain rate test that determines the amount of energy absorbed by a sample during fracture. The absorbed energy is a measure of a given material's toughness. Barcol hardness is used to determine the hardness of both reinforced and non-reinforced rigid plastics. The specimen is placed under the indentor of the Barcol hardness tester and uniform pressure is applied to the specimen until the dial indication reaches a maximum. The depth of the penetration is converted into absolute Barcol numbers.

From Table 3 and Fig. 10 it can be seen that the tensile strength and elongation at break of pure EP are 28.9 MPa and 16.5\% respectively. After adding MWCNTs-COOH, the tensile strength increases to $35.2 \mathrm{MPa}$ and the elongation at break decreases to $12.8 \%$. Moreover, after the addition of various hyperbranched polyester grafting carbon nanotubes, the tensile 
Table 3 Mechanical properties of various EP/MWCNTs- $\mathrm{COOH}$ blends

\begin{tabular}{lllll}
\hline Samples & $\begin{array}{l}\text { Tensile strength } \\
(\mathrm{MPa})\end{array}$ & $\begin{array}{l}\text { Elongation at } \\
\text { break (\%) }\end{array}$ & $\begin{array}{l}\text { Charpy impact } \\
\text { strength }\left(\mathrm{kJ} \mathrm{m}^{-2}\right)\end{array}$ & 4.76 \\
EP & 28.9 & 16.5 & 10.2 & 41.2 \\
EP/MWCNTs-COOH & 35.2 & 12.8 & 12.17 & 48.2 \\
EP/MWCNTs-H201 & 34.3 & 15.9 & 13.24 & 46.1 \\
EP/MWCNTs-H202 & 35.1 & 20.2 & 19.94 & 42.9 \\
EP/MWCNTs-H203 & 36.9 & 24.7 & 21.07 & 30.8 \\
EP/MWCNTs-H204 & 35.8 & 31.3 & &
\end{tabular}

strength have little change compared with MWCNTs-COOH/EP, indicating that the branching degree of hyperbranched polyester has little effect on the tensile strength of EP composites. However, the elongation at break increase obviously.

On the other hand, the Charpy impact strength of MWCNTs$\mathrm{COOH} / \mathrm{EP}\left(10.2 \mathrm{~kJ} \mathrm{~m}^{-2}\right)$ was about $114.3 \%$ higher than that of neat $\mathrm{EP}\left(4.76 \mathrm{~kJ} \mathrm{~m}^{-2}\right)$, while that of various hyperbranched polyester grafting carbon nanotubes and EP composites was further improved. And with the increase of branching degree of hyperbranched polyester, the Charpy impact strength was increased obviously. The Barcol hardness of MWCNTs-COOH/ EP (48.2) was about $17 \%$ higher than that of neat EP (41.2), but compared with MWCNTs-COOH/EP, after the addition of various hyperbranched polyester grafting carbon nanotubes, the Barcol hardness of EP composites decreased significantly, and the higher the branching degree increased, the more obvious the decline of Barcol hardness was.

The above results indicate that the addition of MWCNTs$\mathrm{COOH}$ enhances the toughness of $\mathrm{EP}$, and the further surface grafting of hyperbranched polyester onto MWCNTs-COOH can enhance the toughness of the composites to a larger extent. Two possible mechanisms are proposed: first, a large amount of $-\mathrm{OH}$ in hyperbranched polyester might form hydrogen bond with EP, which improved the interfacial bonding between epoxy resin and carbon nanotubes. And the higher the branching degree of hyperbranched polyester, the stronger the interfacial bonding. Second, the surface grafting enhances the dispersion of carbon nanotubes in the EP matrix.

\subsection{Morphology analysis}

The impact fracture surfaces of pure epoxy resin, carboxylated carbon nanotubes/epoxy resin composites and hyperbranched polyester modified carbon nanotubes/epoxy resin composites were observed by scanning electron microscopy. The addition of carbon nanotubes in each system was $0.3 \%$ of the mass percentage of epoxy resin matrix, as shown in Fig. 11.

From Fig. 11, it can be seen that the dispersion of carboxylated carbon nanotubes in epoxy resin matrix is not uniform, and there is a large area of reunion. Compared with MWCNTs$\mathrm{COOH}$, the area of reunion of MWCNTs-H201 and MWCNTs$\mathrm{H} 202$ decreases and the dispersion improves. As shown in Fig. 11e-h, with the further increase of the degree of branching of hyperbranched polyesters, the samples of MWCNTs-H203/EP

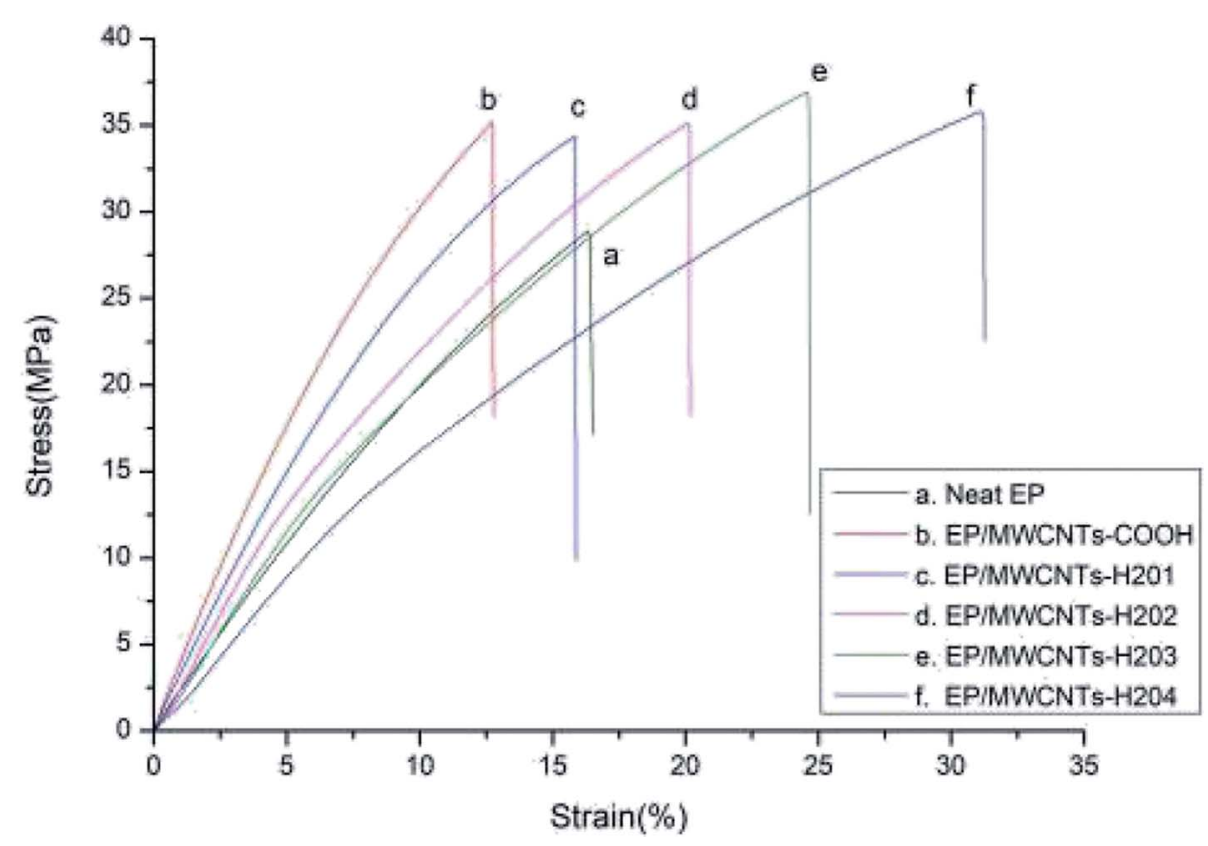

Fig. 10 Tensile diagrams of neat EP and various EP/MWCNTs composites. 


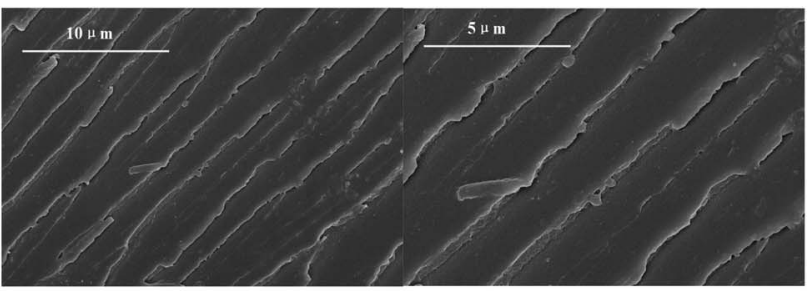

(a)

(b)

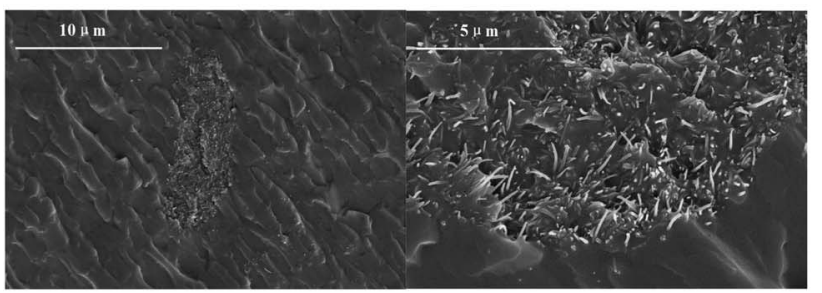

(c)

(d)

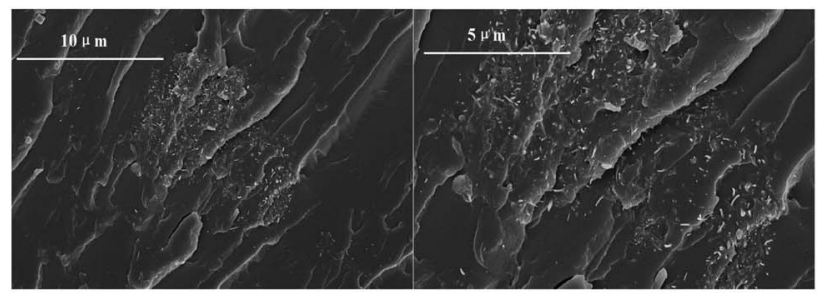

(e)

(f)

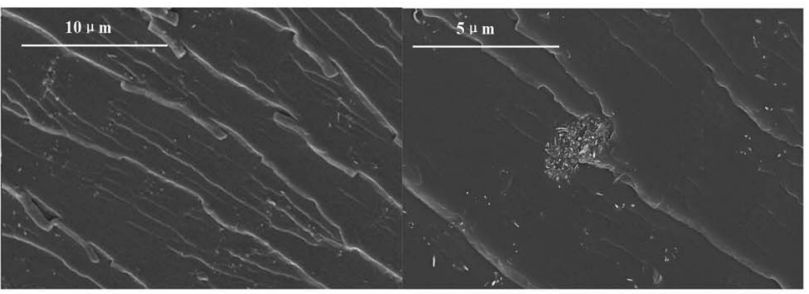

(g)

(h)

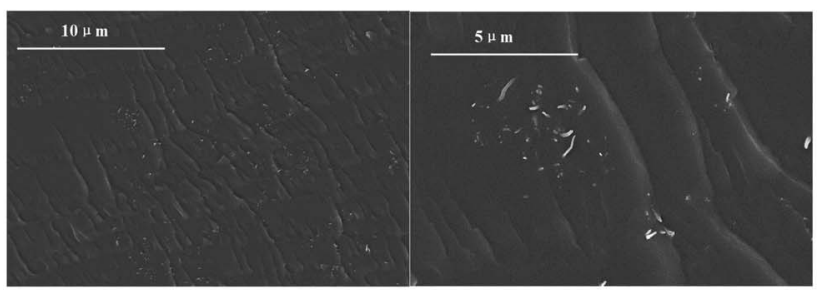

(i)

(j)

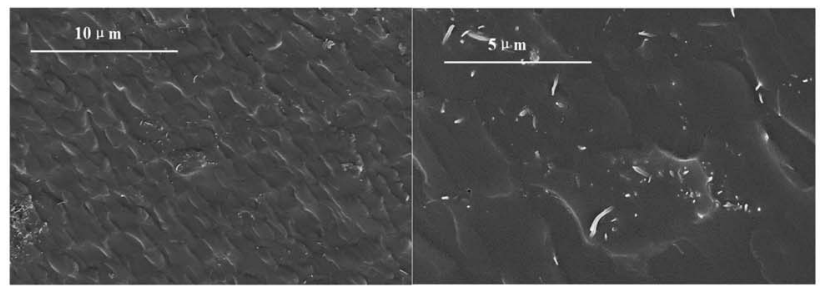

(k)

(l)

Fig. 11 SEM micrographs of fracture surface of different epoxy samples: ( $a$ and b) neat epoxy, (c and d) MWCNTs-COOH/EP, (e and f) MWCNTs-H201/EP, (g and h) MWCNTs-H202/EP, (i and j) MWCNTsH203/EP, (k and l) MWCNTs-H204/EP.

and MWCNTs-H204/EP composites did not find the aggregates of carbon nanotubes, which could basically be considered as nano-scale dispersion.
On the other hand, as shown in Fig. 11a and b, the impact section of pure epoxy resin is smooth, the phase state is uniform, the fracture stripes are straight and layered, and the orientation is orderly, showing typical brittle fracture characteristics. As shown in Fig. 11c and d, after adding $0.3 \%$ mass ratio of MWCNTs-COOH, the fracture surface becomes rough relatively, and the cracks are still linear and partially uniform and orderly. Although there are a few active groups on the surface of MWCNTs-COOH, the bonding energy between MWCNTs-COOH and epoxy resin is weak. When the resin matrix breaks, the carbon nanotubes do not exert too much force on the matrix, resulting in a large number of long striplike ridges on the fracture surface, indicating that the carbon nanotubes are basically pulled out of the resin matrix. ${ }^{\mathbf{4 0 , 4 1}}$ However, with the modification of carbon nanotubes by hyperbranched polyester, the long strip-shaped convex edges on the fracture surface are obviously reduced, as shown in Fig. 11e1 , and with the increase of branching degree, the pull-out phenomenon of carbon nanotubes is less. The surface of MWCNTs-H203 and MWCNTs-H204 are mostly coated with resin matrix materials (Fig. 11i-l), and the exposed part of carbon nanotubes is basically snap rather than pulled out, which indicates that carbon nanotubes and resin matrix form a very strong interface connection, and the higher the branching degree of hyperbranched polymer increases, the stronger the interfacial bonding is. Therefore, when the sample is impacted, the load acting on the resin matrix is transferred to the carbon nanotubes through the interface, which plays a role of bearing load and consuming fracture energy, and prevents the propagation of matrix cracks, greatly improving the strength and toughness of epoxy resin composites.

\section{Conclusions}

The MWCNTs-COOH was modified with hyperbranched polyesters with different molecular structure (different branching degree) by surface graft in the above study. And hyperbranched polyesters with different branching degree were successfully grafted onto MWCNTs-COOH by esterification reaction between the carboxyl groups on the surface of MWCNTs-COOH and the hydroxyl groups of hyperbranched polyester, indicated from the results of FTIR, TEM, XPS, and TGA analysis of various hyperbranched polyesters grafting carbon nanotubes, and proving the higher the branching degree of hyperbranched polyester increased, the higher the grafting rate of carbon nanotubes was. What's more, MWCNTs-COOH and various hyperbranched polyester with different branching degree grafting MWCNTs were added into epoxy prepolymer to prepare the EP/MWCNTs composites respectively, so as to investigate the role of different branching degree hyperbranched polyester surface modification in the mechanical performance of EP/MWCNTs composites. The results of DMA indicated that the addition of MWCNTs-COOH increased the storage modulus and $T_{\mathrm{g}}$ of the pure EP and surface grafting of various hyperbranched polyesters onto MWCNTs-COOH decreased the $T_{\mathrm{g}}$ and loss peak height of the composites. And with the branching degree of hyperbranched polyester increased, the stronger the interfacial 
bonding between MWCNTs and EP matrix. The results of mechanical performance and morphology analysis also revealed that the addition of the surface graft of various hyperbranched polyester onto MWCNTs-COOH significantly improved the dispersion and interfacial bonding in the EP matrix, which exhibited a better performance in the enhancement of toughness of EP. And the higher the branching degree of hyperbranched polyester, the better the toughening performance of EP.

\section{Conflicts of interest}

There are no conflicts to declare.

\section{Acknowledgements}

We gratefully acknowledge the financial support from the National Natural Science Foundation of China (51702282), the Key Industry Technology Innovation Projects of Chongqing (CSTC2017zdcy-zdyf0297), and the Leading Talents of Scientific and Technological Innovation of Chongqing (cstccxljrc201720).

\section{References}

1 M. Qi, Y. Xu, W. Rao, X. Luo, L. Chen and Y. Wang, RSC Adv., 2018, 8, 26948-26958.

2 S. Gao and E. Mäder, Composites, Part A, 2002, 33, 559-576.

3 K. Yang, M. Gu and Y. Jin, J. Appl. Polym. Sci., 2008, 110, 2980-2988.

4 A. Boukerrou, J. Duchet, S. Fellahi and H. Sautereau, J. Appl. Polym. Sci., 2007, 105, 1420-1425.

5 Z. Hao, L. Li, X. Liao, X. Sheng and Y. Zhang, Polym. Bull., 2018, 75, 1013-1026.

6 S. Fatemeh, A. Laura, P. Paola, C. Cosimo, B. Thatiane, C. M. O. Hilário, P. Concetto, S. Filippo and A. Veronica, Polym. Int., 2016, 65, 308-319.

7 W. Li, D. Xiang, L. Wang, E. Harkin-Jones, C. Zhao, B. Wang and Y. Li, RSC Adv., 2018, 8, 26910-26921.

8 L. Lu, L. Xia, H. Zengheng, S. Xingyue, Z. Yi and L. Pan, RSC $A d v .$, 2018, 8, 29830-29839.

9 X. Luo, X. Yu, Y. Ma, K. Naito and Q. Zhang, Thermochim. Acta, 2018, 663, 1-8.

10 W. J. Choi, R. L. Powell and D. S. Kim, Polym. Compos., 2009, 30, 415-421.

11 K. Yang, M. Gu, Y. Jin, G. Mu and X. Pan, Composites, Part A, 2008, 39, 1670-1678.

12 S. Kumar, S. K. Samal, S. Mohanty and S. K. Nayak, Thermochim. Acta, 2017, 654, 112-120.

13 S. Jung-Hoon, P. B. Gangadhara and C. Alan, Polym. Compos., 2017, 38, 1873-1880.

14 A. M. Visco, A. Pistone, V. Brancato, D. Iannazzo and M. Fazio, Polym. Compos., 2016, 37, 1007-1015.

15 T. Tsafack, J. M. Alred, K. E. Wise, B. Jensen, E. Siochi and B. I. Yakobson, Carbon, 2016, 105, 600-606.
16 J. Kang, J. He, Z. Chen, F. Yang, J. Chen, Y. Cao and M. Xiang, Polym. Adv. Technol., 2015, 26, 32-40.

17 J. He, J. Kang, Z. Chen, H. Yu, J. Chen, F. Yang, Y. Cao and M. Xiang, J. Therm. Anal. Calorim., 2015, 119, 1769-1780.

18 J. Wu, J. Guo, Q. Zhang, L. Gao, H. Li, H. Deng, W. Jiang, G. Sui and X. Yang, Polym. Compos., 2018, 39, E733-E744.

19 M. Rajabi, K. Mahanpoor and O. Moradi, RSC Adv., 2017, 7, 47083-47090.

20 R. Castaldo, R. Avolio, M. Cocca, G. Gentile, M. E. Errico, M. Avella, C. Carfagna and V. Ambrogi, RSC Adv., 2017, 7, 6865-6874.

21 A. Visco, A. Pistone, V. Brancato, D. Iannazzo and M. Fazio, Polym. Compos., 2016, 37, 1007-1015.

22 A. Dettlaff, M. Sawczak, E. Klugmann-Radziemska, D. Czylkowski, R. Miotk and M. Wilamowska-Zawłocka, RSC Adv., 2017, 7, 31940-31949.

23 F. Jin, C. Ma and S. Park, Mater. Sci. Eng., 2011, 528, 85178522.

24 Z. Spitalsky, D. Tasis and K. Papagelis, Prog. Polym. Sci., 2010, 35, 357-401.

25 F. Shiravand, L. Ascione, P. Persico, C. Carfagna, T. Brocks, M. O. H. Cioffi, C. Puglisi, F. Samperi and V. Ambrogi, Polym. Int., 2016, 65, 308-319.

26 J. Zhang and C. P. Hu, Eur. Polym. J., 2008, 44, 3708-3714.

27 J. Yang, Z. Chen and G. Yang, Polymer, 2008, 49, 3168-3175.

28 J. Frohlich, H. Kautz and R. Thomann, Polymer, 2004, 45, 2155-2164.

29 B. Xiong, R. Chen, F. Zeng, J. Kang and Y. Men, J. Membr. Sci., 2018, 545, 213-220.

30 W. Han, X. Liao, Q. Yang, G. Li, B. He, W. Zhu and Z. Hao, RSC Adv., 2017, 7, 22515-22523.

31 Z. Hao, Y. Tan, X. Zhang and F. Zhang, J. Wuhan Univ. Technol., Mater. Sci. Ed., 2009, 24, 466-470.

32 F. Zeng, R. Xu, L. Ye, B. Xiong, J. Kang, M. Xiang, L. Li, X. Sheng and Z. Hao, Ind. Eng. Chem. Res., 2019, 58, 22172224.

33 Y. Yu, B. Xiong, F. Zeng, R. Xu, F. Yang, J. Kang, M. Xiang, L. Li, X. Sheng and Z. Hao, Ind. Eng. Chem. Res., 2018, 57, 17142-17151.

34 Y. Yu, F. Zeng, J. Chen, J. Kang, F. Yang, Y. Cao and M. Xiang, Polym. Compos., 2019, 40, E440-E448.

35 Y. Yu, F. Zeng, J. Chen, J. Kang, F. Yang, Y. Cao and M. Xiang, Polym. Int., 2018, 67, 1212-1220.

36 R. Xu, G. Xu, J. Wang, J. Chen, F. Yang, J. Kang and M. Xiang, RSC Adv., 2018, 8, 25236-25247.

37 R. Xu, J. Wang, D. Chen, F. Yang, J. Kang, M. Xiang, L. Li and X. Sheng, RSC Adv., 2018, 8, 41270-41279.

38 J. Wang, R. Xu, F. Yang, J. Kang, Y. Cao and M. Xiang, J. Membr. Sci., 2018, 556, 374-383.

39 D. Ratna and G. Simon, Polymer, 2001, 42, 8833-8839.

40 H. Kim, S. Kim, J. Kim, K. Rhee and J. Kathi, J. Polym. Sci., Part B: Polym. Phys., 2010, 48, 1175-1184.

41 M. Hosur, R. Barua, S. Zainuddin, A. Kumar, J. Trovillion and S. Jeelani, J. Appl. Polym. Sci., 2013, 127, 4211-4224. 